\title{
Reporting of Drug Benefit in FDA-Approved Prescription Drug Labeling
}

\author{
Bansri Desai, PharmD ${ }^{7}$, Kyungwan Hong, PharmD, MS ${ }^{7}$, John H. Powers III, MD², and \\ Peter Doshi, PhD $^{7}$
}

'Department of Pharmaceutical Health Services Research, University of Maryland School of Pharmacy, Baltimore , MD , USA; ' ${ }^{2}$ George Washington University School of Medicine, Washington, DC , USA.

J Gen Intern Med 35(1):377-9

DOI: $10.1007 / \mathrm{s} 11606-019-05460-2$

(c) Society of General Internal Medicine 2019

\section{INTRODUCTION}

Obtaining an accurate understanding of the known benefits and risks of drugs is difficult, particularly when drugs first come to market. The most robust evidence is largely limited to pre-marketing studies (e.g., phase 3 trials), but the peer-reviewed literature describing these studies may be incomplete (e.g., with unpublished trials), and systematic reviews often have yet to be conducted. Despite their reputation as unhelpful, FDA-approved prescribing information (also known as "package inserts" or "drug labels") should provide clinicians with a trustworthy source of information about new drugs that has been independently vetted.

Previous research has assessed the quality of clinical trial evidence supporting recent FDA-approved therapeutic agents ${ }^{1}$ however, how this information is reported to prescribers through drug labels has not been evaluated. Per guidance, labels are supposed to include key efficacy information including the magnitude of treatment benefit, i.e., "the effect that can be attributed to the drug" (measured as the difference between observed effects in new drug and control groups, presented as absolute and/or relative differences), and measures of precision and random error (confidence intervals and $p$ values). ${ }^{2}$ We sought to assess the degree to which labels actually provided this information.

\section{METHODS}

Original drug labels for all new molecular entities (NMEs) approved by the FDA ${ }^{3}$ between January 2015 and March 2018 were downloaded from the Drugs@FDA online database. We excluded NMEs not treating or preventing diseases or conditions (e.g., diagnostic products) and not tested in controlled trials (e.g., single-arm

Published online October 28, 2019 studies). Text, tables, and figures in each label's "Clinical Studies" section were independently evaluated by two authors, and disagreements were resolved through consensus with an additional author. For each indication, we extracted the primary efficacy outcome (either explicitly stated or implicitly defined) and recorded the presence or absence of the following attributes describing treatment effects for primary outcomes: point estimates, confidence intervals, and $p$ values. Primary efficacy outcomes were categorized as either a surrogate endpoint or direct measure of patient benefit. We classified presentation of treatment effects as absolute and relative differences and whether results for new drug and control groups were presented separately. Drug therapeutic class was based on the American Hospital Formulary Service classification. $^{4}$

\section{RESULTS}

One hundred thirty indications (from 119 NMEs) were approved between January 2015 and March 2018. One hundred two indications met inclusion criteria. Forty-five percent (46/ 102) of primary outcomes were surrogate endpoints, not direct measures of patient benefit. Some endpoints (e.g., "clinical cure") were listed but without a specific definition. Twentyfive percent (25/102) of indications included comparisons against active controls, $71 \%$ (72/102) against non-active controls, and 5\% (5/102) against both active and non-active controls.

Drug labels for 78 (76\%) indications provided point estimates of drugs' treatment effects, and $73(72 \%)$ and $55(54 \%)$ also reported confidence intervals and $p$ values, respectively (Table 1$)$. Labels for 24 (25\%) indications reported no estimates of treatment effect. Data were most often presented in tables and not in text (i.e., sentence format).

Completeness of data reported varied by therapeutic area. Dermatology and infectious disease drug labels were least likely, and cancer and cardiovascular drugs were most likely to report treatment differences along with 
Table 1 Description of Treatment Effects in FDA-Approved Labels

\begin{tabular}{|c|c|c|c|c|}
\hline & \multicolumn{4}{|c|}{ No. (\%) } \\
\hline & Text & Table & Figure & $\begin{array}{l}\text { Anywhere ( } n \\
=102)\end{array}$ \\
\hline $\begin{array}{l}\text { Any statement of } \\
\text { treatment efficacy }\end{array}$ & $\begin{array}{l}66 \\
(65)\end{array}$ & $\begin{array}{l}91 \\
(89)\end{array}$ & $14(14)$ & $100(98)$ \\
\hline Point estimate & $\begin{array}{l}20 \\
(30)\end{array}$ & $\begin{array}{l}70 \\
(77)\end{array}$ & $\begin{array}{l}14 \\
(100)\end{array}$ & $78(76)$ \\
\hline Confidence interval & $\begin{array}{l}15 \\
(23)\end{array}$ & $\begin{array}{l}66 \\
(73)\end{array}$ & $7(50)$ & $73(72)$ \\
\hline$p$ value & $\begin{array}{l}14 \\
(21)\end{array}$ & $\begin{array}{l}46 \\
(51)\end{array}$ & $5(36)$ & $55(54)$ \\
\hline $\begin{array}{l}\text { Point estimate, } \\
\text { confidence interval and } \\
p \text { value }\end{array}$ & $\begin{array}{l}10 \\
(15)\end{array}$ & $\begin{array}{l}35 \\
(38)\end{array}$ & $5(36)$ & $45(44)$ \\
\hline
\end{tabular}

${ }^{a}$ Any statement indicating efficacy of the drug. This includes statements without numerical estimates and point estimates of individual treatment groups

measures of precision (Table 2). Most labels (92\%) reported separate point estimates for new drug and control groups, more often than the difference between the groups.

\section{DISCUSSION}

Prescription drug labels are intended to provide clinicians with information necessary to support use of interventions in clinical practice. However, we found substantial variability in reporting of treatment effects. Although most labels reported results by individual study groups, the goal of clinical trials is a comparison between groups with associated precision. Overall, one in four lacked a measure of treatment effect (the difference between the groups) and a similar proportion omitted confidence intervals for these estimates.

Labels not reporting estimates of treatment effects and associated precision can mislead clinicians when interpreting net benefit versus harms of treatments. Surveys of clinicians and patients indicate that both frequently hold inaccurate expectations, overestimating benefits and underestimating harms. ${ }^{5,} 6$ Information about drugs' treatment effects should be reported clearly and consistently for drug labeling to fulfill its stated purpose, including treatment effects and the precision of those effects.

Data Sharing: All data we extracted and coded, as well as copies of original drug labels used in our study, are freely available in the Zenodo repository (https://doi.org/10.5281/ zenodo.2562183).

Corresponding Author: Peter Doshi, PhD; Department of Pharmaceutical Health Services Research University of Maryland School of Pharmacy, Baltimore, MD , USA (e-mail: pdoshi@rx.umaryland.edu).

Author Contributions All authors had full access to all of the data in the study and take responsibility of the integrity of the data and the accuracy of the data analysis.

Study concept and design: Desai, Doshi 
Acquisition of data: All authors

Analysis and interpretation of data: All authors

Drafting of the manuscript: Desai, Doshi, Powers

Critical revision of the manuscript for important intellectual content:

All authors

Statistical analysis: Desai

Administrative, technical, or material support: Doshi

Study supervision: Doshi

Funding Information This study was funded with nonspecific faculty research funds provided by the University of Maryland, Baltimore, to Dr. Doshi.

\section{Compliance with Ethical Standards:}

Conflict of Interest: Dr. Doshi has received travel funds from the European Respiratory Society (2012) and Uppsala Monitoring Center (2018) and grants from the Laura and John Arnold Foundation (20172021), American Association of Colleges of Pharmacy (2015), PatientCentered Outcomes Research Institute (2014-2016), Cochrane Methods Innovations Fund (2016-2018), and UK National Institute for Health Research (2011-2014) and is an editor at The BMJ and an unpaid member of the Reagan-Udall Foundation for the FDA. Dr. Powers reports personal fees from Abbvie, Cardeas, Cempra, Contrafect, Gilead, Johnson \& Johnson, MedImmune, Novartis, Otsuka, Pfizer, Roche, Romark, and Trius outside the submitted work. Dr. Desai and Dr. Hong have no interests to declare.

\section{REFERENCES}

1. Downing NS, Aminawung JA, Shah ND, Krumholz HM, Ross JS Clinical Trial Evidence Supporting FDA Approval of Novel Therapeutic Agents, 2005-2012. JAMA. 2014;311(4):368-377. doi:https://doi.org/10. 1001/jama.2013.282034

2. Clinical Studies Section of Labeling for Human Prescription Drug and Biological Products-Content and Format. Rockville, MD: U.S. Food and Drug Administration; 2006:25. https://www.fda.gov/media/72140/ download. Accessed 17 Sept 2019.

3. Food and Drug Administration. New Drugs at FDA: CDER's New Molecular Entities and New Therapeutic Biological Products. https://www.fda.gov/ Drugs/DevelopmentApprovalProcess/DrugInnovation/default.htm. Accessed 17 Sept 2019

4. AHFS Classification - Drug Assignments and Reassignments. http://ahfs ashp.org/drug-assignments.aspx. Accessed 17 Sept 2019.

5. Hoffmann TC, Del Mar C. Clinicians' Expectations of the Benefits and Harms of Treatments, Screening, and Tests: A Systematic Review. JAMA Intern Med 2017;177(3):407-419. doi:https://doi.org/10.1001/ jamainternmed.2016.8254

6. Hoffmann TC, Del Mar C. Patients' expectations of the benefits and harms of treatments, screening, and tests: a systematic review. JAMA Intern Med 2015;175(2):274-286. doi:https://doi.org/10.1001/jamainternmed.2014. 6016

Publisher's Note Springer Nature remains neutral with regard to jurisdictional claims in published maps and institutional affiliations. 